\title{
Can $\alpha$-radioimmunotherapy increase efficacy for the systemic control of cancer?
}

\begin{abstract}
"Disadvantages lie in the reluctance of some nuclear medicine departments to be involved with $\alpha$-emitting radioisotopes, perhaps because of the uncertainty in the dosimetry and the inability to readily determine whether isolated cells in transit are eliminated."
\end{abstract}

\section{KEYWORDS: $\alpha$-dosimetry $\approx \alpha$-radiation $\approx$ Ac:Bi generator cell clusters $\approx$ isolated cancer cells $\approx$ radioimmunotherapy $\approx$ radioisotopes $\approx$ tumors}

Since the development of the hybridoma [1], monoclonal antibodies (mAbs) have been raised against many antigens overexpressed by cancer and other cells [2]. Most of these mAbs are benign with little antibody-dependent cell-mediated cytotoxicity (ADCC), caused by lysis of antibody-coated target cells by effector cells with cytolytic activity and Fc receptors. Cell-mediated cytotoxicity arises from cytolysis of a target cell by effector lymphocytes, such as cytotoxic $\mathrm{T}$ lymphocytes or NK cells and may be antibody-dependent or -independent. Another limitation relates to the expression of the targeted antigens being only on a subset of cancer cells. However, some antibodies work by neutralizing or blocking receptors, and these tend to be the more effective.

The following antigens and mAbs have been approved by the US FDA for clinical use:

- ErbB: HER1/EGF receptor (cetuximab and panitumumab);

- HER2/neu (trastuzumab);

- EpCAM (catumaxomab and edrecolomab);

- VEGF-A (bevacizumab);

- Lymphoid: CD20 (ibritumomab, ofatumumab, rituximab and tositumomab);

- CD52(alemtuzumab);

- Myeloid: CD33 (gemtuzumab).

The major therapeutic antibodies on the market are Avastin $^{\circledR}$ (Roche, Basel, Switzerland) and Herceptin $^{\circledR}$ (Genentech, CA, USA; both oncology), Humira ${ }^{\circledR}$ (Abbott, IL, USA) and Remicade ${ }^{\circledR}$ (Centocor, PA, USA; both autoimmune and infectious disease) and Rituxan ${ }^{\circledR}$ (Genentech, CA, USA; oncology and autoimmune and infectious disease), and accounted for $80 \%$ of revenues in 2006.
Benign or partial blocking $\mathrm{mAbs}$ that are cancer cell specific can still be effective if labeled with a toxin. The $\mathrm{mAb}$ then becomes a targeting vector to transport the toxin to the targeted cancer cells. In such cases, the half-life of the mAb in the body should preferably match the half-life of the toxin. Toxins can be chemicals or radioisotopes and are mostly chelated to the $\mathrm{mAb}$ to form relatively stable immunoconjugates. Chemical toxins can have long half-lifes in the body (e.g., ricin), which increases their toxicity for normal tissue. Radioisotopes have both a wide range of half-lifes and radiation decay properties.

Nuclear imaging uses long lived $\gamma$-emitters, allowing blood clearance as the tumor increases its uptake of the conjugate over time, thus improving contrast. ${ }^{131} \mathrm{I}$ and ${ }^{123} \mathrm{I},{ }^{201} \mathrm{Tl},{ }^{67} \mathrm{Ga}$ and ${ }^{111} \mathrm{In}$ are some reactor- and cyclotron-produced radioisotopes for this purpose. $\beta$-emitting radioisotopes, predominantly ${ }^{131} \mathrm{I}$, are used for therapy. The radioisotopes are generally conjugated with a bifunctional chelator attached to the targeting antibody to form the radio-immunoconjugate (IC). However, the early success of $\beta$-emitting radioimmunotherapy has been modest [2].

In recent times, high linear energy transfer (LET) radiation in the form of auger electrons and $\alpha$-particles have been studied. Auger and Koster-Kronig electron transitions cause the emission of multiple, low-energy and very short-range electrons, such that the LET can still be high. However, the short range requires access into the nucleus to cause single-strand breaks (SSBs) and double-strand breaks (DSBs) in the DNA. $\alpha$-particles have ranges from 20 to $80 \mu \mathrm{m}$ and the radioisotope sources can be located on cell membranes or nearby cells. The LET is, typically, approximately $100 \mathrm{keV} / \mu \mathrm{m}$ with a high probability of causing DSBs (although the ratio SSB/DSB is $\sim 20$, compared with $\sim 60$ for low LET radiation) [3]. $\alpha$-radiation is ideal for killing

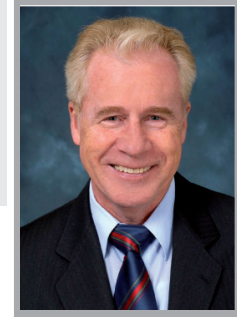

Barry J Allen Centre for Experimental Radiation Oncology, St George Cancer Care Centre, Gray Street, Kogarah, NSW 217, Australia Tel.: +61291133855 Fax: +61291134044 barry.allen@sesiahs.health. nsw.gov.au 
isolated cancer cells in transit in the vascular and lymphatic systems, and for inducing tumor regression by killing tumor capillary endothelial cells. Apoptosis is the dominant form of cell death with high LET radiation [4]. A programmed sequence of events leads to the elimination of cells without releasing harmful substances into the surrounding area. Apoptosis plays a crucial role in developing and maintaining health by eliminating old, unnecessary and unhealthy cells.

\section{Therapeutic objectives}

Traditionally, cancer management begins with surgery to remove solid tumors, radiotherapy to reduce local recurrence (as in breast cancer) and chemotherapy, to control systemic disease or for palliation. Immunotherapy is a developing approach that could make a major contribution to the management of cancer.

There are three separate objectives that must be addressed and each objective requires a different approach:

- Kill isolated cancer cells in transit in the lymphatic and vascular circulation;

- Regress prevascular and lymphatic lesions;

- Regress tumor vasculature and tumors.

\section{Isolated cancer cells}

These cells have particular problems relating to cell cycle and bioavailability:

- Cells may be outside the cell cycle in the G0 phase. As such, they are relatively insensitive to radiotherapy and chemotherapy;

- Systemic cancer targeting is required, but only a small fraction of the dose will reach its target so short range cytotoxic action is essential to reduce normal tissue damage, which will be the dose-limiting factor;

- High labeling efficiency is required to reduce saturation of targeted antigens by unlabelled mAbs;

- Short half-life is preferred as cells in the vascular system can be reached quickly. The long half-life of chemotoxins in the body is not indicated for such targets. As such, $\alpha$-radiation is best suited for the toxic agent;

- Lymphatic administration may be essential to eliminate cells in transit from primary lesions;

- The therapeutic response is difficult to determine. However, magnetic cell separation of cancer cells in the peripheral blood with the magnetic microspheres coated with the targeting mAbs could solve this problem [5].

\section{Cell clusters}

Cell clusters occur when the cell cycle is switched on in the appropriate seed/soil environment. However, endothelial cell growth factors, expressed by cancer cells, are still insufficient to stimulate the vascular extensions into the lesion.

Clusters can only be reached by nonvascular transport. Long-range cross-fire, as for $\beta$-emitters, is not indicated but useful short-range cross-fire can occur for $\alpha$-emitters [6].

Chemical toxins have no cross-fire effect and would be much less effective in cell clusters with limited penetration. The long half-life of chemotoxins in the body is not indicated for such targets.

\section{Tumors}

Tumor capillary permeability is an important parameter that determines, in part, the bioavailabilty of the cancer cells in a tumor. Leaky neogenic capillaries allow the extravasation of the IC into the perivascular space to saturate the targeted antigens expressed by contiguous and adjacent cancer cells.

Long-range cross-fire effect gives $\beta$-radiation an important advantage and reduces the effect of heterogenous uptake of the $\beta$-IC in the tumor.

Intralesional injections with $\alpha$-ICs can overcome this problem but may not be indicated for systemic disease.

Chemotoxins will suffer from bioavailability and the lack of a cross-fire effect.

\section{" $\alpha$-therapy needs, first, to establish maximum tolerance doses for practical acceptance."}

The potential role of the bystander effect [7] could be of benefit here. This radiation-induced phenomenon causes unirradiated cells to exhibit effects as a result of signals received from nearby irradiated cells, causing a mutation in the nucleus of the hit cells. Cells that are not directly hit by an $\alpha$-particle, but are in the vicinity of one that is hit, also contribute to the genotoxic response of the cell population. Similarly, when the medium containing irradiated cells is transferred to unirradiated cells, these cells show bystander responses when assayed for clonogenic survival and oncogenic transformation.

Tumor capillary permeability causes a high density of IC-labeled antigens around the capillaries, with a rapid drop off with distance from the capillaries. Whereas this is a drawback for $\beta$-emitting ICs, it enhances the toxicity of $\alpha$-ICs to the capillary endothelial cells. The short 
range $(\sim 80 \mu \mathrm{m})$ of the emitted $\alpha$-radiation ensures a high radiation dose to the endothelial cell nucleus, inducing apoptosis. The capillary will close and if enough such capillaries close down, the tumor will regress. This process is called tumor antivascular $\alpha$-therapy (TAVAT) and explains the tumor regressions observed in the Phase I clinical trial of systemic targeted $\alpha$-therapy (TAT) of metastatic melanoma [8] . This process could be assisted by tumor vascular disruption agents that increase tumor capillary permeability.

"The demonstration of the synergy between tumor vascular disruption agents and [tumor antivascular $\alpha$-therapy] could bring about a sea of change in cancer therapy."

\section{Ac:Bi generator}

Therapies must be practical in their application. The $\alpha$-emitting lanthanide, ${ }^{149} \mathrm{~Tb}$, was produced at the CERN (Organisation Européenne pour la Recherche Nucléaire [Switzerland]) GeV synchrotron in therapeutic quantities [9] but was not a practical solution. ${ }^{211}$ At is produced by a 30-MeV cyclotron [10] but the 7-h half-life limits is application outside the production center. This is not the case for the ${ }^{225} \mathrm{Ac}:{ }^{213} \mathrm{Bi}$ generator as the mother isotope has a 10-day half-life, allowing distribution around the world. ${ }^{225} \mathrm{Ac}$ is separated from ${ }^{229} \mathrm{Th}$ or produced by a $30-\mathrm{MeV}$ cyclotron [11]. ${ }^{213} \mathrm{Bi}$ is eluted and used to prepare the $\alpha$-IC [12]. The half-life of $46 \mathrm{~min}$ is quite adequate for this purpose. Thus, the Ac:Bi generator for therapy could become the equivalent of the Mo:Tc generator for imaging. ${ }^{225} \mathrm{Ac}$ has also been studied as a therapeutic agent because it is highly cytotoxic with four $\alpha$-particles emitted per decay [13].

\section{$\alpha$-dosimetry}

In $\beta$-therapy, the Medical Internal Radiation Dose method can be used to determine the radiation absorbed doses in the target and other organs. This is not the case for $\alpha$-radiation as the actual location of the emitter determines the dose delivered on the microscopic scale. Monte Carlo calculations are needed with the precise location and structure of the target and normal tissue cells. Real-time dosimetry may be achievable with MOSFET (metal-oxide semiconductor field-effect transistor) microdosimeters implanted into the blood vessels. However, off-line biological dosimetry can be implemented by inducing damaged lymphocytes to divide and form micronuclei in the mitotic state. These micronuclei result from DSBs in the DNA, which form discrete entities of bilayer phospholipid-enclosed DNA fragments within the lymphocyte prior to division [14].

\section{Current status of targeted $\alpha$-therapy Status quo}

There are a number of past and current Phase I clinical trials of TAT for cancer [15]. These are for acute myeloid leukemia at the Memorial SloanKettering Cancer Center (NY, USA) [16], metastatic melanoma at St George Hospital, (Sydney, Australia) [17,18], gliomas [10,19], lymphoma [20] and ovarian cancer [21].

\section{Strengths}

The advantages of TAT are that:

- Patients do not need to be isolated post-treatment;

- For liquid cancers, such as acute myelogenous leukemia, the uptake plateaus in approximately $5 \mathrm{~min}$ in the leukemia volumes;

- Tumor antivascular $\alpha$-therapy can be effective well below the maximum tolerance dose in solid tumors;

- The Ac generator can be readily disposed of because of its short, 10-day half-life.

\section{Weaknesses}

Disadvantages lie in the reluctance of some nuclear medicine departments to be involved with $\alpha$-emitting radioisotopes, perhaps because of the uncertainty in the dosimetry and the inability to readily determine whether isolated cells in transit are eliminated. The last two problems could be resolved with biological dosimetry and magnetic cell separation of cancer cells in the blood with the magnetic microspheres coated with the targeting mAbs.

\section{The way forward}

$\alpha$-therapy needs, first, to establish maximum tolerance doses for practical acceptance. This has been determined with ${ }^{213} \mathrm{Bi}$-IC only for acute myelogenous leukemia at approximately $1 \mathrm{mCi} / \mathrm{kg}$ [16] The maximum tolerance dose has not yet been established for metastatic melanoma, but the efficacious dose for some melanomas is certainly less than $0.3 \mathrm{mCi} / \mathrm{kg}$ [18] and for intracavity glioblastoma multiforme (GBM) it is approximately $0.14 \mathrm{mCi} / \mathrm{kg}$ for ${ }^{211} \mathrm{At}-\mathrm{IC}$ [10].

The next stage is to determine the efficacy in Phase II trials at the MTD or the effective dose. A current study of combined modalities for acute 
myelogenous leukemia is ongoing at Memorial Sloan-Kettering Cancer Center using chemotherapy to reduce the cancer load followed by ${ }^{213} \mathrm{Bi}-\mathrm{IC}$ to further reduce the cancer.

The melanoma trial showed significant efficacy in the Phase I trial, achieving 10\% near complete or partial response, $40 \%$ stable disease and $13 \%$ long-term survival of 2-5 years, without any evidence of adverse events [17,18]. The Duke GBM study achieved a median survival of 52 weeks for GBM [10].

While these results are relatively impressive, the potential of TAT to reduce solid tumors by tumor antivascular $\alpha$-therapy remains just that.
The demonstration of the synergy between tumor vascular disruption agents and TAVAT could bring about a sea of change in cancer therapy.

\section{Financial \& competing interests disclosure}

The author has no relevant affiliations or financial involvement with any organization or entity with a financial interest in or financial conflict with the subject matter or materials discussed in the manuscript. This includes employment, consultancies, honoraria, stock ownership or options, expert testimony, grants or patents received or pending, or royalties.

No writing assistance was utilized in the production of this manuscript.

16 Jurcic GJ, Larson SM, Sgouros G et al: Targeted $\alpha$ particle immunotherapy for myeloid leukaemia. Blood 100, 1233-1239 (2002).

17 Raja C, Graham P, Rizvi SMA et al.: Interim analysis of toxicity and response in Phase 1 trial of systemic targeted $\alpha$ therapy for metastatic melanoma. Canc. Biol. Ther. 6(6), 846-852 (2007).

18 Allen BJ, Raja C, Rizvi SMA, Graham P, Kearsley JH: New directions for clinical trials of targeted $\alpha$ therapy for metastatic melanoma. Curr. Radio-Pharmaceut. 1, 240-250 (2008)

19 Cordier D, Forrer F, Bruchertseifer F et al.: Targeted $\alpha$-radionuclide therapy of functionally critically located gliomas with ${ }^{213} \mathrm{Bi}$-DOTA-[Thi8,Met $\left.(\mathrm{O} 2) 11\right]$-substance $\mathrm{P}$ : a pilot trial. Eur. J. Nucl. Med. Mol. Imaging 37, 1335-1344 (2010).

20 Schmidt D, Neumann F, Antke C et al.: Phase 1 Clinical Study on $\alpha$-therapy for Non-Hodgkin Lymphoma. In: 4th AlphaImmunotherapy Symposium. Morgenstern A, (Ed.). Institute for Transuranium Elements, Dusseldorf, Germany, 27-29 June 2004.

14 Song EY, Rizvi SMA, Raja C et al.: The cytokinesis-block assay as a biological dosimeter for targeted $\alpha$ therapy. Phys. Med. Biol. 53, 319-328 (2008).

15 Allen BJ: Clinical trials of targeted $\alpha$ therapy for cancer. Rev. Rec. Clin. Trials 3, 185-191 (2008).
21 Andersson H, Cederkrantz E, Back T et al.: Intraperitoneal $\alpha$-particle radioimmunotheapy of ovafrian cancer patients: pharmacokinetics and dosimetry of ${ }^{211}$ AT-MX35F $\left(\mathrm{ab}^{\prime}\right)_{2}$ - a Phase I study. J. Nucl. Med. 50 (7), 1153-1160 (2009). a mechanism for the regression of solid tumours in metastatic cancer. Phys. Med. Biol. 52, L15-L19 (2007). 